REFERENCES

[1] T. E. Djaferis, "Finite frequency tests for robust performance of systems with real parameter uncertainty," IEEE Trans. Automat. Contr., vol. AC-36, no. 12, pp. 1477-1482, 1991.

[2] R. A. Frazer and W. J. Duncan, "On the criteria for stability for small motions," in Proc. Royal Society A, vol. 124, pp. 642-654, 1929.

[3] L. Qiu and E. J. Davison, "A simple procedure for the exact stability robustness computation of polynomials with affine coefficient perturbations," Syst. Contr. Lett., vol. 13, pp. 413-420, 1989.

[4] R. T. Rockafellar, Convex Analysis. Princeton, NJ: Princeton Univ., 1970.

\section{Sampled-Data Observers with Generalized Holds for Unstable Plants}

Wassim M. Haddad, Hsing-Hsin Huang, and Dennis S. Bernstein

\begin{abstract}
Optimality conditions are derived for reduced-order sampled-data estimation satisfying an observation constraint that involves a prespecified, possibly unstable, subspace of the system dynamics. It is shown that a generalized hold device possessing a time-varying exponential intersample profile is essential in order to account for the unstable dynamics. In particular, it is shown that with a continuous-time quadratic performance measure a zero-order-hold device may result in an unbounded (infinite) cost. An additional feature of the problem is the utilization of an averaging $\mathbf{A} / \mathrm{D}$ device within the continuous-time/discrete-time conversion which results in averaged measurements depending upon delayed samples of the state.
\end{abstract}

\section{INTRODUCTION}

Due to advances in digital computers, discrete-time estimators for continuous-time systems have been developed and used in numerous applications. It is well known that optimal discrete-time estimates of the dynamic states of a continuous-time model are given by a discretetime Kalman filter, which is based on an equivalent discrete-time model. Closer inspection of the effects of sampling and reconstruction between continuous- and discrete-time operations, however, revelas subtleties beyond "naive" (that is, not sampled-data) discrete-time estimation theory for an equivalent discrete-time model. Three issues need to be addressed, namely, measurements, system dynamics, and performance measure.

The sampling operation, which requires careful treatment of noisy measurements, can have ramifications in applying standard sampleddata estimation theory [1]-[3]. Since it is meaningless to sample white noise, instantaneous $A / D$ devices cannot be used in the presence of noisy measurements. Consequently, we utilize an averaging-type $\mathrm{A} / \mathrm{D}$ device of the form

$$
\hat{y}(k) \triangleq \frac{1}{t_{k}-t_{k-1}} \int_{t_{k-1}}^{t_{k}} y(t) d t
$$

Manuscript received May 18, 1992; November 17, 1992. This work was supported in part by the Air Force Office of Scientific Research under gran F49620-92-J-0127 and the National Science Foundation under grant ECS9109558.

W. M. Haddad and H.-H. Huang are with the Department of Mechanical and Aerospace Engineering, Florida Institute of Technology, Melbourne, FL 32901.

D. S. Bernstein is with the Department of Aerospace Engineering, The University of Michigan, Ann Arbor, MI 48109-2140.

IEEE Log Number 9213578 where

$$
y(t)=C x(t)+w(t)
$$

and $w(\cdot)$ is a continuous-time white noise process. The discretetime measurement now involves a discretized noise signal whose properties need to be accounted for. Specifically, the resulting averaged measurements depend upon delayed samples of the state [1], [2]. In this case, the equivalent discrete-time model can be captured by a suitably augmented system involving nonnoisy (discrete-time) measurements. The above technique was proposed in [4] and utilized in [1]-[3], [5], [6].

In practice, it is often necessary to obtain estimators for plants possessing unstable dynamics [7]-[10]. This leads to the consideration of a generalized architecture for the digital filter. Specifically, it is shown (see Remark 2.3) that if a standard zero-order-hold reconstruction device is used in the presence of unstable dynamics, then the equivalent discrete-time least squares performance criterion is unbounded. This problem can be circumvented by employing a generalized hold device possessing a time-varying exponential intersample profile. Hence, we show that a time-varying reconstruction architecture is essential for sampled-data estimation of unstable plants. In practice, standard procedures for designing sampled-data estimators for unstable plants involve extrapolation of the estimator output between measurements [11, pp. 116-119]. The results given herein can thus be viewed as a formalization of this procedure. In the context of feedback control, generalized hold devices were extensively developed in [12] to achieve a variety of effects such as simultaneous pole placement and decoupling. Related results that exploit time-varying effects can be found in [13]-[15].

Along with the above-mentioned points, the evaluation of the leastsquares performance criterion for sampled-data estimation requirs special attention. Specifically, the cost functional involves intersample behavior and leads to a discrete-time performance criterion having a highly complex structure [1]. It should be noted that the resulting discrete-time cost does not possess the familiar least-squares structure as in the naive discrete-time estimation problem.

Finally, constraints on implementation complexity often make it desirable in practice to design estimators of reduced order. Such loworder estimators are also motivated by the fact that although a system model may hlave many degrees of freedom (such as coloring filter states and vibrational modes), it is often the case that estimates of only a small number of state variables (e.g., rigid body position and rotational modes) are actually required. Hence, in the present note, we derive optimality conditions for reduced-order sampled-data observerestimators in which the observation subspace of the estimator is constrained a priori to include all of the unstable modes and selected stable modes. Hence, the results presented herein generalize the results of $[7,8]$ to the sampled-data setting.

\section{NOTATION}

$\begin{array}{ll}I_{r}, 0_{r \times s}, 0_{r} & r \times r \text { identity matrix, } r \times s \text { zero matrix, } r \times r \text { zero } \\ & \text { matrix. } \\ ()^{T}, \mathrm{tr} & \text { Transpose, trace. } \\ \mathbb{E}, \mathbb{R}, \mathbb{R}^{r \times s} & \text { Expected value, real numbers, } r \times s \text { real matrices. } \\ n, l, n_{e}, q & \text { Positive integers, } 1 \leq n_{e} \leq n+l . \\ x, y, x_{e}, y_{e} & n, l, n_{e}, q \text {-dimensional vectors. } \\ A, C & n \times n, l \times n \text { matrices. } \\ A_{e}, B_{e}, C_{e}, D_{e} & n_{e} \times n_{e}, n_{e} \times l, q \times n_{e}, q \times l \text { matrices. }\end{array}$




$\begin{array}{ll}w_{1}, w_{2} & n, l \text {-dimensional zero-mean continuous-time white } \\ & \text { noise processes. } \\ V_{1} & n \times n \text { nonnegative-definite intensity of } w_{1} \\ V_{2} & l \times l \text { positive-definite intensity of } w_{2} \\ V_{12} & n \times l \text { cross intensity of } w_{1}, w_{2} \\ R & q \times q \text { positive-definite matrix. } \\ L & q \times n \text { matrix. } \\ t, k & t \in[0, \infty), \text { discrete-time index, } 1,2,3, \cdots\end{array}$

III. Sampled-Data Subspace-ObSerVER PRoblemand EQUIVALENT DISCRETE-TIME FORMULATION

In this section, we state the fixed-order sampled-data subspaceobserver problem. In the problem formulation the sample interval $h$ and the estimator order $n_{e}$ are a priori fixed and the optimization is performed with respect to the estimator parameters.

Fixed-Order, Sampled-Data Subspace-Observer Problem: Given the $n$ th-order continuous-time system

$$
\dot{x}(t)=A x(t)+w_{1}(t), \quad t \in[0, \infty),
$$

with $\mathrm{A} / \mathrm{D}$ averaged measurements

$$
\hat{y}(k h) \triangleq \frac{1}{h} \int_{(k-1) h}^{k h} y(t) d t, \quad k=1,2,3, \cdots,
$$

where

$$
y(t)=C x(t)+w_{2}(t),
$$

design an $n_{e}$ th-order observer

$$
x_{e}(k+1)=A_{e} x_{e}(k)+B_{e} \hat{y}(k h),
$$

with D/A generalized hold output

$$
y_{e}(k h+s)=C_{e}(s) x_{e}(k)+D_{e}(s) \hat{y}(k h), \quad s \in[0, h],
$$

which satisfies the following design criteria:

1. the observer (2.4), (2.5) is a steady-state asymptotic observer for a specified $n_{u}$-dimensional subspace of the plant (2.1) where $n_{e}=n_{u}+l ;$ and

2. the observer is an optimal estimator that minimizes the leastsquares state-estimation error criterion

$$
\begin{array}{r}
\mathcal{T}\left(A_{e}, B_{e}, C_{e}(\cdot), D_{e}(\cdot)\right) \triangleq \lim _{t \rightarrow \infty} \mathbb{E} \frac{1}{t} \int_{0}^{t}\left[L x(s)-y_{e}(s)\right]^{T} R \\
\cdot\left[L x(s)-y_{e}(s)\right] d s .
\end{array}
$$

To enforce the first condition, partition (2.1) and (2.3) according to

$$
\begin{aligned}
x(t) & =\left[\begin{array}{l}
x_{u}(t) \\
x_{s}(t)
\end{array}\right] \in \mathbb{R}^{n}, \quad x_{u}(t) \in \mathbb{R}^{n_{u}}, \quad x_{s}(t) \in \mathbb{R}^{n_{s}}, \\
{\left[\begin{array}{l}
\dot{x}_{u}(t) \\
\dot{x}_{s}(t)
\end{array}\right] } & =\left[\begin{array}{cc}
A_{u} & A_{u s} \\
0_{n_{s} \times n_{u}} & A_{s}
\end{array}\right]\left[\begin{array}{l}
x_{u}(t) \\
x_{s}(t)
\end{array}\right]+\left[\begin{array}{c}
w_{1 u}(t) \\
w_{1 s}(t)
\end{array}\right] \\
y(t) & =\left[\begin{array}{ll}
C_{u} & C_{s}
\end{array}\right]\left[\begin{array}{l}
x_{u}(t) \\
x_{s}(t)
\end{array}\right]+w_{2}(t) .
\end{aligned}
$$

The partitioned form of the plant dynamics $A$ appearing in (2.8) allows us to characterize the two subspaces corresponding to $x_{u}$ and $x_{s}$. The $n_{s} \times n_{u}$ zero matrix in the lower-left block of $A$ is needed to achieve asymptotic observation of $x_{u}$ independently of $x_{s}$. If necessary, the plant dynamics $A$ can be recast in the form (2.8) by using a similarity transformation to a modal basis. The coupling matrix $A_{u s}$ can be either zero or nonzero. Furthermore, we assume that $A_{s}$ is asymptotically stable. Finally, the matrix $L$, which is partitioned as $L \triangleq\left[\begin{array}{ll}L_{u} & L_{s}\end{array}\right]$, where $L_{u}$ and $L_{s}$ are $q \times n_{u}$ and $q \times n_{s}$ matrices, identifies the states or linear combinations of states whose estimates are desired.
Finally, as will be shown, in order to account for the unstable dynamics it is necessary to implement an observer with generalized hold outputs as characterized by the functions $C_{e}(\cdot)$ and $D_{e}(\cdot)$ which are time-varying over the sample interval $[0, h]$. As shown below, $C_{e}(\cdot)$ is chosen to ensure that $\mathcal{T}$ is finite while $D_{e}(\cdot)$ is optimized in order to minimize the least-squares state-esimation error criterion (2.6).

The first result of this section concerns the propagation of the plant and the discretized measurements over one time step. For notational convenience in stating the main result define

$$
H(s) \triangleq \int_{0}^{s} e^{A r} d r .
$$

Theorem 2.1: For the fixed-order, subspace-observer problem, the plant dynamics (2.8), averaged measurements (2.9), and least-squares observation-error criterion (2.6) have the equivalent discrete-time representations

$$
\begin{gathered}
x((k+1) h)=A^{\prime} x(k h)+w_{1}^{\prime}(k h), \\
\hat{y}(k h)=C^{\prime} x((k-1) h)+w_{2}^{\prime}((k-1) h), \\
\mathcal{T}\left(A_{e}, B_{e}, C_{e}(\cdot), D_{e}(\cdot)\right) \\
\triangleq \delta+\lim _{k \rightarrow \infty} \mathbb{E} \frac{1}{h} \int_{0}^{h}\left\{\left[L e^{A s} x(k h)-y_{e}(k h+s)\right]^{T}\right. \\
\left.\cdot R\left[L e^{A s} x(k h)-y_{e}(k h+s)\right]\right\} d s,
\end{gathered}
$$

where

$$
\begin{gathered}
A^{\prime} \triangleq e^{A h} \\
C^{\prime} \triangleq \frac{1}{h} C H(h) \\
w_{1}^{\prime}(k h) \triangleq \int_{0}^{h} e^{A(h-s)} w_{1}(k h+s) d s \\
w_{2}^{\prime}((k-1) h) \triangleq \frac{1}{h} C \int_{0}^{h} \int_{0}^{s} e^{A(s-r)} w_{1}[(k-1) h+r] d r d s \\
+\frac{1}{h} \int_{0}^{h} w_{2}[(k-1) h+s] d s \\
\delta \triangleq \frac{1}{h} \operatorname{tr} \int_{0}^{h} \int_{0}^{s} e^{A r} V_{1} e^{A^{T} r} L^{T} R L d r d s
\end{gathered}
$$

and $w_{1}^{\prime}(k h)$ and $w_{2}^{\prime}(k h)$ are zero-mean, white noise processes with

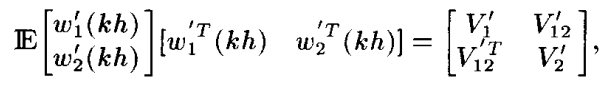

where

$$
\begin{gathered}
V_{1}^{\prime}=\int_{0}^{h} e^{A s} V_{1} e^{A^{T s}} d s \\
V_{12}^{\prime}=\frac{1}{h} \int_{0}^{h} e^{A s} V_{1} H^{T}(s) d s C^{T}+\frac{1}{h} H(h) V_{12}, \\
V_{2}^{\prime}=\frac{1}{h} V_{2}+\frac{1}{h^{2}} C \int_{0}^{h} H(s) V_{1} H^{T}(s) d s C^{T} \\
+\frac{1}{h^{2}} V_{12}^{T} \int_{0}^{h} H^{T}(s) d s C^{T}+\frac{1}{h^{2}} C \int_{0}^{h} H(s) d s V_{12} .
\end{gathered}
$$

Proof: The proof of this theorem is a lengthy but straightforward calculation involving integrals of white noise signals, and hence is omitted. 
Remark 2.I: Note that the equivalent discrete-time least squares estimation error criterion involves a constant positive offset $\delta$ which serves as a lower bound on the sampled-data performance due to the discretization process.

Next, note that due to the upper-block-triangular structure of $A$ in (2.8), the equivalent discrete-time dynamics matrix $e^{A h}$ will also have an upper-block-triangular structure. Thus, in accordance with our earlier partitioning, (2.10) and (2.11) can be represented as

$$
\begin{aligned}
& {\left[\begin{array}{l}
x_{u}((k+1) h) \\
x_{s}((k+1) h)
\end{array}\right]=\left[\begin{array}{cc}
A_{u}^{\prime} & A_{u s}^{\prime} \\
0_{n_{s} \times n_{u}} & A_{s}^{\prime}
\end{array}\right]\left[\begin{array}{l}
x_{u}(k h) \\
x_{s}(k h)
\end{array}\right]+\left[\begin{array}{l}
w_{1 u}^{\prime}(k h) \\
w_{1 s}^{\prime}(k h)
\end{array}\right]} \\
& \hat{y}(k h)=\left[\begin{array}{ll}
C_{u}^{\prime} & C_{s}^{\prime}
\end{array}\right]\left[\begin{array}{l}
x_{u}((k-1) h) \\
x_{s}((k-1) h)
\end{array}\right]+w_{2}^{\prime}((k-1) h),
\end{aligned}
$$

where

$$
A_{u}^{\prime} \triangleq e^{A_{u} h}, \quad A_{s}^{\prime} \triangleq e^{A_{s} h}, \quad A_{u s}^{\prime} \triangleq \int_{0}^{h} e^{A_{u}(h-r)} A_{u s} e^{A_{s} r} d r .
$$

Note that (2.11), or, equivalently, (2.19), shows that the averaged measurements depend upon delayed samples of the state. Thus, by augmenting the discretized state equation (2.10) to include these measurements and preserving the partitioned form given by (2.18) and (2.19), it is possible to represent the original sampled-data problem as a discrete-time problem involving nonnoisy measurements.

Corollary 2.1: With the notation

$$
\begin{aligned}
& \hat{x}_{u}(k h) \triangleq\left[\begin{array}{c}
x_{u}(k h) \\
\hat{y}(k h)
\end{array}\right], \quad \hat{x}(k h) \triangleq\left[\begin{array}{l}
\hat{x}_{u}(k h) \\
x_{s}(k h)
\end{array}\right], \\
& \hat{A}_{u} \triangleq\left[\begin{array}{cc}
A_{u}^{\prime} & 0_{n_{u} \times l} \\
C_{u}^{\prime} & 0_{l}
\end{array}\right], \quad \hat{A}_{u s} \triangleq\left[\begin{array}{c}
A_{u s}^{\prime} \\
C_{s}^{\prime}
\end{array}\right] \\
& \hat{A} \triangleq\left[\begin{array}{cc}
\hat{A}_{u} & \hat{A}_{u s} \\
0_{n_{s} \times\left(n_{u}+l\right)} & A_{s}^{\prime}
\end{array}\right], \\
& \hat{C}_{u} \triangleq\left[0_{l \times n_{u}} \quad I_{l}\right], \quad \hat{C} \triangleq\left[\begin{array}{ll}
\hat{C}_{u} & 0_{l \times n_{s}}
\end{array}\right] \\
& \hat{w}_{1 u}(k h) \triangleq\left[\begin{array}{c}
w_{1 u}^{\prime}(k h) \\
w_{2}^{\prime}(k h)
\end{array}\right], \quad \hat{w}(k h) \triangleq\left[\begin{array}{c}
\hat{w}_{1 u}(k h) \\
w_{1 s}^{\prime}(k h)
\end{array}\right], \\
& \hat{V} \triangleq \mathbb{E}\left[\hat{w}(k h) \hat{w}^{T}(k h)\right], \\
& \hat{L}(s) \triangleq\left[L_{u} e^{A_{u} s} 0_{q \times l} L_{u} \int_{0}^{s} e^{A_{u}(s-r)} A_{u s} e^{A_{s} r} d r+L_{s} e^{A_{s} s}\right], \\
& s \in[0, h],
\end{aligned}
$$

the fixed-order sampled-data subspace-observer problem is equivalent to the following discrete-time problem. Given the $(n+l)$ th-order discrete-time system

$$
\hat{x}((k+1) h)=\hat{A} \hat{x}(k h)+\hat{w}(k h),
$$

with discrete-time nonnoisy measurements

$$
\hat{y}(k h)=\hat{C} \hat{x}(k h),
$$

design an $n_{e}$ th-order observer of the form (2.4), (2.5) that minimizes

$$
\begin{aligned}
& \mathcal{T}\left(A_{e}, B_{e}, C_{e}(\cdot), D_{e}(\cdot)\right) \\
& \triangleq \delta+\lim _{k \rightarrow \infty} \mathbb{E} \frac{1}{h} \int_{0}^{h}\left\{\left[\hat{L}(s) \hat{x}(k h)-y_{e}(k h+s)\right]^{T}\right. \\
& \left.\cdot R\left[\hat{L}(s) \hat{x}(k h)-y_{e}(k h+s)\right]\right\} d s .
\end{aligned}
$$

Remark 2.2: Note that the measurements $\hat{y}(k h)$ are noise free. However, due to the discrete-time setting this singularity is not as serious as singular measurement noise in a continuous-time setting where the Kalman filter gains are expressed in terms of the inverse of the measurement noise intensity. However, as in nonstrictly proper continuous-time estimation with nonnoisy measurements this formulation leads to a static projection matrix defined below. (See [16], [17] for details.)

Next, to enforce the observation constraint 1), define the error states

$$
z_{u}(k h) \triangleq \hat{x}_{u}(k h)-x_{e}(k),
$$

and require that

$$
\lim _{k \rightarrow \infty} z_{u}(k h)=0,
$$

for all $x(0)$ and $x_{e}(0)$ when $w_{1}(t) \equiv 0$, and $w_{2}(t) \equiv 0$. The requirement (2.24) implies that zero asymptotic observation error for a specified $\left(n_{u}+l\right)$-dimensional subspace is achieved under zero external disturbances and arbitrary initial conditions. Thus, the goal of the reduced-order subspace-observer problem is to design a reduced-order observer of order $n_{e}=n_{u}+l$ that observes a specified plant subspace and provides optimal estimation of a specified linear combination of states. Note that the observation constraint (2.24) can be satisfied even if the subspace corresponding to $\hat{x}_{u}$ is unstable [7]-[9]. Thus we allow $\hat{A}_{u}$ to possess unstable as well as stable modes. Of course, the results presented herein remain valid if $\hat{A}_{u}$ is asymptotically stable. The subscript $u$, however, reminds us that $\hat{A}_{u}$ is permitted to be unstable. However, since we require that $A_{s}$ be continuous-time asymptotically stable this implies that $A_{s}^{\prime}$ is discretetime asymptotically stable. In applications, the matrix $A_{s}^{\prime}$ may include the dynamics of all coloring filter states as well as damped vibrational modes.

Before continuing it is useful to point out that in the full-order case $n_{u}=n$ and hence $n_{e}=n+l$. In this case the observer/estimator can observe all of $\hat{x}(k h)=\left[x^{T}(k h) \hat{y}^{T}(k h)\right]^{T}$. Note that the increase in plant order from $n$ to $n+l$ is due to the A/D process. Hence, in this context an $n$ th-order observer can be regarded as being of reduced order.

It now folows from (2.3) and (2.20) that

$$
\begin{aligned}
z_{u}((k+1) h)= & \left(\hat{A}_{u}-B_{e} \hat{C}_{u}\right) \hat{x}_{u}(k h) \\
& -A_{e} x_{e}(k)+\hat{A}_{u s} x_{s}(k)+\hat{w}_{1 u}(k h) .
\end{aligned}
$$

At this point we make the crucial observation that the explicit dependence of the error states $z_{u}(k h)$ on the states $\hat{x}_{u}(k h)$ (containing unstable modes) can be eliminated by constraining

$$
A_{e}=\hat{A}_{u}-B_{e} \hat{C}_{u}
$$

so that

$$
z_{u}((k+1) h)=\left(\hat{A}_{u}-B_{\epsilon} \hat{C}_{u}\right) z_{u}(k h)+\hat{A}_{u s} x_{s}(k h)+\hat{w}_{1 u}(k h) .
$$

Now using the constraint (2.25) it follows from (2.20) and (2.26) that the augmented equivalent discrete-time system is given by

$$
\tilde{x}((k+1) h)=\tilde{A} \tilde{x}(k h)+\hat{w}(k h),
$$

where

$$
\tilde{x}(k h) \triangleq\left[\begin{array}{l}
z_{u}(k h) \\
x_{s}(k h)
\end{array}\right], \quad \tilde{A} \triangleq \hat{A}-\left[\begin{array}{c}
I_{n_{u}+l} \\
0
\end{array}\right] B_{e} \hat{C} .
$$

Lemma 2.1: $\tilde{A}$ is discrete-time asymptotically stable if and only if $A_{e}$ is discrete-time asymptotically stable. 
Proof: The proof follows by simply noting that

$$
\tilde{A}=\left[\begin{array}{cc}
A_{e} & \hat{A}_{u s} \\
0_{n_{s} \times\left(n_{u}+l\right)} & A_{s}^{\prime}
\end{array}\right]
$$

is upper block triangular and $A_{s}^{\prime}$ is asymptotically stable.

Next, we show that the explicit dependence of the cost functional on the unstable dynamics can be eliminated by employing a special choice of the generalized hold function $C_{e}(\cdot)$. To motivate this choice write (2.22) as:

$$
\begin{aligned}
& \mathcal{T}\left(A_{e}, B_{e}, C_{e}(\cdot), D_{e}(\cdot)\right) \\
&=\delta+\lim _{k \rightarrow \infty} \mathbb{E} \frac{1}{h} \int_{0}^{h}\left\{\left[\left(\hat{L}(s)-D_{e}(s) \hat{C}\right) \hat{x}(k h)\right.\right. \\
&\left.-C_{e}(s) x_{e}(k)\right]^{T} R\left[\left(\hat{L}(s)-D_{e}(s) \hat{C}\right) \hat{x}(k h)\right. \\
&\left.\left.-C_{e}(s) x_{e}(k)\right]\right\} d s,
\end{aligned}
$$

or, equivalently,

$$
\begin{aligned}
& \mathcal{T}\left(A_{e}, B_{e}, C_{e}(\cdot), D_{e}(\cdot)\right) \\
&= \delta+\lim _{k \rightarrow \infty} \mathbb{E} \frac{1}{h} \int_{0}^{h}\left\{\left[\left(\left[L_{u} e^{A_{u} s} 0\right]-D_{e}(s) \hat{C}_{u}\right) z_{u}(k h)\right.\right. \\
&+\left[\left(\left[L_{u} e^{A_{u} s} 0\right]-D_{e}(s) \hat{C}_{u}\right)-C_{e}(s)\right] x_{e}(k) \\
&\left.+\left(L_{u} \int_{0}^{s} e^{A_{u}(s-r)} A_{u s} e^{A_{s} r} d r+L_{s} e^{A_{s} s}\right) x_{s}(k h)\right]^{T} R \\
& \cdot\left[\left(\left[L_{u} e^{A_{u} s} 0\right]-D_{e}(s) \hat{C}_{u}\right) z_{u}(k h)+\left[\left(\left[L_{u} e^{A_{u} s} 0\right]\right.\right.\right. \\
&\left.\left.-D_{e}(s) \hat{C}_{u}\right)-C_{e}(s)\right] x_{e}(k) \\
&\left.\left.+\left(L_{u} \int_{0}^{s} e^{A_{u}(s-r)} A_{u s} e^{A_{s} r} d r+L_{s} e^{A_{s} s}\right) x_{s}(k h)\right]\right\} d s .
\end{aligned}
$$

Now note that the dependence of $\mathcal{T}$ on $\hat{x}_{u}$ (or, equivalently, $x_{e}$ which may contain unstable states) can be eliminated by constraining

$$
C_{e}(s)=\left[\begin{array}{ll}
L_{u} e^{A_{u} s} & 0_{q \times l}
\end{array}\right]-D_{e}(s) \hat{C}_{u}, \quad s \in[0, h] .
$$

With this choice the cost can now be expressed in terms of the augmented second-moment matrix. First, however, to guarantee that $\mathcal{T}$ is finite, consider the set of asymptotically stable reduced-order estimators

$\mathcal{S} \triangleq\left\{\left(A_{e}, B_{e}, C_{e}(\cdot), D_{e}(\cdot)\right): A_{e}=\hat{A}_{u}-B_{e} \hat{C}_{u}\right.$ is discrete-time

asymptotically stable $\}$

so that $\tilde{A}$ is discrete-time asymptotically stable. Of course, $\mathcal{S}$ is nonempty if $\left(\hat{A}_{u}, \hat{C}_{u}\right)$ is detectable.

Proposition 2.1: The equivalent discrete-time cost functional (2.22) is finite if $\dot{C}_{\epsilon}(\cdot)$ is given by $(2.28)$. In this case $(2.22)$ is given by

$$
\mathcal{T}\left(A_{e}, B_{e}, C_{e}(\cdot), D_{e}(\cdot)\right)=\delta+\operatorname{tr} Q \tilde{R},
$$

where the steady-state covariance

$$
Q \triangleq \lim _{k \rightarrow \infty} \mathbb{E}\left[\tilde{x}(k h) \tilde{x}^{T}(k h)\right]
$$

exists and satisfies the algebraic Lyapunov equation

$$
Q=\tilde{A} Q \tilde{A}^{T}+\hat{V}
$$

where

$$
\tilde{R} \triangleq \frac{1}{h} \int_{0}^{h}\left[\hat{L}(s)-D_{e}(s) \hat{C}\right]^{T} R\left[\hat{L}(s)-D_{e}(s) \hat{C}\right] d s .
$$

Proof: For simplicity of exposition, in the first part of the proof we assume that $n_{e}=n+l$ and $D_{e}(s)=0, s \in[0, h]$. The proof of the more general case is similar but requires more extensive algebraic manipulations. Assuming $C_{e}(s)=\left[L e^{A s} 0_{q \times i}\right], s \in[0, h]$, it follows from (2.27) that

$$
\begin{aligned}
\mathcal{T}\left(A_{e}, B_{e}, C_{e}(\cdot)\right) \\
=\delta+\lim _{k \rightarrow \infty} \mathbb{E} \frac{1}{h} \int_{0}^{h}\left\{\left[L e^{A s} 0\right] \hat{x}(k h)-C_{e}(s) x_{e}(k)\right\}^{T} \\
\quad \cdot R\left\{\left[L e^{A s} 0\right] \hat{x}(k h)-C_{e}(s) x_{e}(k)\right\} d s,
\end{aligned}
$$

or, equivalently,

$$
\begin{array}{r}
\mathcal{T}\left(A_{e}, B_{e}, C_{e}(\cdot)\right)=\delta+\lim _{k \rightarrow \infty} \mathbb{E} \frac{1}{h} \int_{0}^{h} z^{T}(k h)\left[L e^{A s} 0\right]^{T} \\
\cdot R\left[L e^{A s} 0\right] z(k h) d s,
\end{array}
$$

where $z(k h)=\hat{x}(k h)-x_{e}(k)$. Now, since $\left(A_{e}, B_{e}, C_{e}(\cdot)\right) \in \mathcal{S}$ it follows that $\lim _{k \rightarrow \infty} Q(k h)$ exists and $\mathcal{T}\left(A_{e}, B_{e}, C_{e}(\cdot)\right)$ is finite. Finally, assuming $\left(A_{e}, B_{e}, C_{e}(\cdot), D_{e}(\cdot)\right) \in \mathcal{S}$ it follows from Lemma 2.1 that $\tilde{A}$ is asymptotically stable. Now the second-moment equation (2.31) is immediate (see [18, p. 471]), while (2.29) follows by direct verification.

Remark 2.3: As mentioned in the introduction the generalized hold function $C_{e}(\cdot)$ posses a time-varying exponential intersample profile. To see this, assume for simplicity that $n_{e}=n+l$ and $D_{e}=0$. In this case, it follows from (2.28) that

$$
C_{e}(s)=\left[\begin{array}{ll}
L e^{A s} & 0_{q \times l}
\end{array}\right], \quad s \in[0, h] .
$$

Alternatively, if we use a D/A zero-order-hold estimate $y_{e}(t)=$ $C_{e} x_{e}(k), t \in[k h,(k+1) h]$, with $C_{e}=\left[L 0_{q \times l}\right]$, then (2.22) becomes

$$
\begin{aligned}
\mathcal{T}\left(A_{e}, B_{e}, C_{e}\right)= & \delta \\
& +\lim _{k \rightarrow \infty} \mathbb{E} \frac{1}{h} \int_{0}^{h}\left\{\left[L e^{A s} 0\right] \hat{x}(k h)\right. \\
& \left.-[L 0] x_{e}(k)\right\}^{T} R\left\{\left[L e^{A s} 0\right] \hat{x}(k h)\right. \\
& \left.-[L 0] x_{e}(k)\right\} d s,
\end{aligned}
$$

or, equivalently,

$$
\begin{aligned}
\mathcal{T}\left(A_{e}, B_{e}, C_{e}\right)= & \delta+\lim _{k \rightarrow \infty} \mathbb{E} \frac{1}{h} \int_{0}^{h}\left\{\left[e^{A s} 0\right] z(k h)\right. \\
& \left.-\left[\left(e^{A s}-I\right) 0\right] x_{e}(k)\right\}^{T} L^{T} R L \\
& \cdot\left\{\left[e^{A s} 0\right] z(k h)-\left[\left(e^{A s}-I\right) 0\right] x_{e}(k)\right\} d s .
\end{aligned}
$$

Note that in this case the explicit dependence of the unstable modes contained within $\hat{x}(k h)$ or, equivalently, $x_{e}(k)$ may drive $\mathcal{T}(\cdot)$ to infinity as $k \rightarrow \infty$.

Next, we present a simple example that explicitly shows that employing a D/A zero-order-hold reconstruction architecture may lead to an unbounded state estimation error criterion. Specifically, we consider a rigid body revolving about an axis of symmetry with mass moment of inertia $I=1$ under a standard white noise disturbance. The system dynamics is modeled as a simple inertia, 
i.e., $I \ddot{\theta}(t)=w(t)$, so that the state-space model is given by

$$
\left[\begin{array}{l}
\dot{x}_{1}(t) \\
\dot{x}_{2}(t)
\end{array}\right]=\left[\begin{array}{ll}
0 & 1 \\
0 & 0
\end{array}\right]\left[\begin{array}{l}
x_{1}(t) \\
x_{2}(t)
\end{array}\right]+\left[\begin{array}{l}
0 \\
1
\end{array}\right] w(t)
$$

Next, assuming perfect estimation, i.e., $x_{e}(k)=x(k h), L=R=I_{2}$, and $n_{e}=n$ it follows from (2.27) with $C_{e}(s)=L=I_{2}$ and $D_{e}(s)=0, s \in[0, h]$, that

$$
\begin{aligned}
\mathcal{T}\left(A_{e}, B_{e}, C_{e}\right)= & \delta+\lim _{k \rightarrow \infty} \mathbb{E} \frac{1}{h} \\
& \cdot \int_{0}^{h}\left[e^{A s} z(k h)+\left(e^{A s}-I_{2}\right) x_{e}(k)\right]^{T} \\
& \cdot\left[e^{A s} z(k h)+\left(e^{A s}-I_{2}\right) x_{e}(k)\right] d s,
\end{aligned}
$$

where $A=\left[\begin{array}{ll}0 & 1 \\ 0 & 0\end{array}\right]$. Next, noting that $e^{A h}=\left[\begin{array}{ll}1 & h \\ 0 & 1\end{array}\right]$ and assuming $x(0)=\left[\begin{array}{l}0 \\ 0\end{array}\right]$ it follows from (2.37) that

$$
\mathcal{T}\left(A_{e}, B_{e}, C_{e}\right)=\delta+\lim _{k \rightarrow \infty} \frac{h^{2}}{3} \mathbb{E}\left[x_{2}(k h)\right]^{2} .
$$

Finally, computing the $(2,2)$ element of the solution to $Q(k h)=$ $\mathbb{E}\left[x(k h) x^{T}(k h)\right]$ given by

$$
Q((k+1) h)=e^{A h} Q(k h) e^{A^{T} h}+V^{\prime},
$$

where

$$
V^{\prime}=\int_{0}^{h} e^{A s} V_{1} e^{A^{T} s} d s=\left[\begin{array}{cc}
h^{3} / 3 & h^{2} / 2 \\
h^{2} / 2 & h
\end{array}\right],
$$

we obtain

$$
Q_{2}((k+1) h)=Q_{2}(k h)+h, \quad k=1,2,3, \cdots .
$$

Noting that (2.39) implies $Q_{2}(k h)=k h$ and that $\mathbb{E}\left[x_{2}(k h)\right]^{2}=$ $Q_{2}(k h)$ it follows from (2.38) that $\mathcal{T}$ is unbounded. Alternatively, using a generalized hold architecture, it follows from (2.27) that in this case

$$
\mathcal{T}\left(A_{\epsilon}, B_{e}, C_{e}(\cdot)\right)=\delta+\lim _{k \rightarrow \infty} \mathbb{E} \frac{1}{h} \int_{0}^{h} z^{T}(k h) e^{A^{T} s} e^{A s} z(k h) d s .
$$

Now, noting that $z(k h)=0$ since $x_{e}(k)=x(k h)$, we obtain $\mathcal{T}\left(A_{e}, B_{e}, C_{e}(\cdot)\right)=\delta$ which is clearly finite.

\section{NECESSARY CONDITIONS FOR THE}

\section{EquivalentDiscrete-Time Problem}

In this section, we obtain necesssary conditions for optimality that characterize solutions to the optimal reduced-order sampleddata subspace-observer problem. For nondegeneracy we restrict our attention to the set of admissible estimators

$$
\mathcal{S}^{\prime} \triangleq\left\{\left(A_{e}, B_{e}, C_{e}(\cdot), D_{e}(\cdot)\right) \in \mathcal{S}:\left(A_{e}, C_{e}(\cdot)\right)\right.
$$

is completely observable over the sample interval $\}$.

Theorem 3.1: If $\left(A_{e}, B_{e}, C_{e}(\cdot), D_{e}(\cdot)\right) \in \mathcal{S}^{\prime}$ solves the optimal sampled-data reduced-order subspace-observer problem with constraints (2.25) and (2.28), then there exist $(n+l) \times(n+l)$ nonnegative-definite matrices $Q$ and $P$ such that

$$
\begin{gathered}
A_{e}=\Phi \hat{A} \nu_{\perp} F^{T}, \\
B_{e}=\Phi \hat{A} Q \hat{C}^{T}\left(\hat{C} Q \hat{C}^{T}\right)^{-1}, \\
C_{e}(s)=\left[\hat{L}(s)-D_{e}(s) \hat{C}\right] F^{T}, \quad s \in[0, h], \\
D_{e}(s)=\hat{L}(s) Q \hat{C}^{T}\left(\hat{C} Q \hat{C}^{T}\right)^{-1}, \quad s \in[0, h],
\end{gathered}
$$

and such that $Q$ and $P$ satisfy

$$
\begin{gathered}
Q=\hat{A} Q \hat{A}^{T}+\hat{V}-\hat{A} \nu Q \nu^{T} \hat{A}^{T}+\mu_{\perp} \hat{A} \nu Q \nu^{T} \hat{A}^{T} \mu_{\perp}^{T}, \\
P=(\hat{A}-\mu \hat{A} \nu)^{T} P(\hat{A}-\mu \hat{A} \nu)+\tilde{R},
\end{gathered}
$$

where

$$
\begin{gathered}
P \triangleq\left[\begin{array}{cc}
P_{u} & P_{u s} \\
P_{u s}^{T} & P_{s}
\end{array}\right] \in \mathbb{R}^{\left(n_{u}+l+n_{s}\right) \times\left(n_{u}+l+n_{s}\right)}, \quad P_{u}>0 \\
F \triangleq\left[\begin{array}{lll}
I_{n_{u}+l} & 0_{\left(n_{u}+l\right) \times n_{s}}
\end{array}\right], \quad \Phi \triangleq\left[\begin{array}{ll}
I_{n_{u}+l} & P_{u}^{-1} P_{u s}
\end{array}\right] \\
\mu \triangleq F^{T} \Phi=\left[\begin{array}{cc}
I_{n_{u}+l} & P_{u}^{-1} P_{u s} \\
0_{n_{s} \times\left(n_{u}+l\right)} & 0_{n_{s}}
\end{array}\right], \quad \mu_{\perp} \triangleq I_{n+l}-\mu, \\
\nu \triangleq Q \hat{C}^{T}\left(\hat{C} Q \hat{C}^{T}\right)^{-1} \hat{C}, \quad \nu_{\perp} \triangleq I_{n+l}-\nu .
\end{gathered}
$$

Furthermore, the minimal cost is given by (2.29). Conversely, if there exist $(n+l) \times(n+l)$ nonnegative-definite $Q$ and $P$ satisfying (3.5) and (3.6), and such that $\hat{C} Q \hat{C}^{T}>0$, then $Q$ satisfies (2.31) with $\left(A_{e}, B_{e}, C_{e}(\cdot), D_{e}(\cdot)\right)$ given by (3.1)-(3.4). Furthermore, $\left(\tilde{A}, \tilde{V}^{1 / 2}\right)$ is stabilizable if and only if $A_{e}$ is asymptotically stable. In this case $\left(A_{e}, C_{e}(\cdot)\right)$ is completely observable over the sample interval.

Proof: The proof is similar to the proof given in [7] and hence is omitted.

Remark 3.1: Note that with $B_{e}$ given by (3.2), the expressions (3.1) and (3.3) for $A_{e}$ and $C_{e}$ are equivalent to the constraints (2.25) and (2.28).

Remark 3.2: Note that the gains $C_{e}(\cdot)$ and $D_{e}(\cdot)$ are matrix functions over the finite interval $[0, h]$. This of course is consistent with our generalized hold architecture.

Next, we specialize Theorem 3.1 to the full-order case $n_{u}=n+l$. As discussed in [7], [8], this case corresponds to $\Phi=F=\mu=I_{n+l}$ without loss of generality. To develop further connections with standard Kalman filter theory, we also assume

$$
D_{e}(s)=0, \quad s \in[0, h] .
$$

Corollary 3.1: Let $n_{e}=n+l$, assume (3.11) is satisfied and suppose $\left(A_{e}, B_{e}, C_{e}(\cdot)\right)$ solves the optimal sampled-data reducedorder subspace-observer problem. Then there exists $(n+l) \times(n+l)$ nonnegative-definite matrix $Q$ such that

$$
\begin{gathered}
A_{e}=\hat{A} \nu_{\perp}, \\
B_{e}=\hat{A} Q \hat{C}^{T}\left(\hat{C} Q \hat{C}^{T}\right)^{-1}, \\
C_{e}(s)=\left[\begin{array}{ll}
L e^{A s} & 0_{q \times l}
\end{array}\right], \quad s \in[0, h],
\end{gathered}
$$

where $Q$ satisfies

$$
Q=\hat{A} Q \hat{A}^{T}-\hat{A} \nu Q \nu^{T} \hat{A}^{T}+\hat{V} .
$$

Furthermore, the minimal cost is given by

$$
\mathcal{T}\left(A_{e}, B_{e}, C_{e}(\cdot)\right)=\delta+\operatorname{tr} Q \hat{R},
$$

where

$$
\hat{R}=\frac{1}{h} \int_{0}^{h}\left[\begin{array}{ll}
L e^{A s} & 0_{q \times l}
\end{array}\right]^{T} R\left[\begin{array}{ll}
L e^{A s} & 0_{q \times l}
\end{array}\right] d s .
$$




\section{NUMERICAL EVALUATION OF INTEgrals INVOLVINGMATRIX EXPONENTIALS}

To evaluate the exponential/integral expressions appearing in Theorem 2.1 , we utilize the approach given by [19]. This approach eliminates the need for integration by computing the matrix exponential of appropirate block matrices. For details on numerical matrix exponentiation see [20]. For simplicity of exposition, we assume $D_{e}=0$.

Proposition 4.1: Consider the following partitioned matrix exponentials of order $(3 n+l) \times(3 n+l)$ and $(3 n) \times(3 n)$, respectively:

$$
\begin{aligned}
& {\left[\begin{array}{cccc}
F_{1} & F_{2} & F_{3} & F_{4} \\
0_{n} & F_{5} & F_{6} & F_{7} \\
0_{n} & 0_{n} & F_{8} & F_{9} \\
0_{l \times n} & 0_{l \times n} & 0_{l \times n} & I_{l}
\end{array}\right] \triangleq \exp \left[\begin{array}{cccc}
-A & I_{n} & 0_{n} & 0_{n \times l} \\
0_{n} & -A & V_{1} & V_{12} \\
0_{n} & 0_{n} & A^{T} & C^{T} \\
0_{l \times n} & 0_{l \times n} & 0_{l \times n} & 0_{l}
\end{array}\right] h,} \\
& {\left[\begin{array}{ccc}
F_{10} & F_{11} & F_{12} \\
0_{n} & F_{13} & F_{14} \\
0_{n} & 0_{n} & F_{15}
\end{array}\right] \triangleq \exp \left[\begin{array}{ccc}
-A & I_{n} & 0_{n} \\
0_{n} & -A & V_{1} \\
0_{n} & 0_{n} & A
\end{array}\right] h,} \\
& {\left[\begin{array}{cccc}
F_{16} & F_{17} & F_{18} & F_{19} \\
0_{n} & F_{20} & F_{21} & F_{22} \\
0_{n} & 0_{n} & F_{23} & F_{24} \\
0_{l \times n} & 0_{l \times n} & 0_{l \times n} & I_{l}
\end{array}\right] \triangleq \exp \left[\begin{array}{cccc}
-A & I_{n} & 0_{n} & 0_{n \times l} \\
0_{n} & -A & V_{1} & 0_{n \times l} \\
0_{n} & 0_{n} & A^{T} & C^{T} \\
0_{l \times n} & 0_{l \times n} & 0_{l \times n} & 0_{l}
\end{array}\right] h,} \\
& {\left[\begin{array}{ccc}
F_{25} & F_{26} & F_{27} \\
0_{n} & F_{28} & F_{29} \\
0_{n} & 0_{n} & F_{30}
\end{array}\right] \triangleq \exp \left[\begin{array}{ccc}
-A^{T} & I_{n} & 0_{n} \\
0_{n} & -A^{T} & L^{T} R L \\
0_{n} & 0_{n} & A
\end{array}\right] h .}
\end{aligned}
$$

Then

$$
\begin{gathered}
A^{\prime}=F_{8}^{T}, \quad C^{\prime}=\frac{1}{h} F_{9}^{T}, \quad \delta=\frac{1}{h} \operatorname{tr}\left(L^{T} R L F_{15}^{T} F_{12}\right), \\
V_{1}^{\prime}=F_{8}^{T} F_{6}, \quad V_{12}^{\prime}=\frac{1}{h} F_{8}^{T} F_{7}, \\
V_{2}^{\prime}=\frac{1}{h}\left(V_{2}+\frac{1}{h} C F_{8}^{T} F_{4}+\frac{1}{h} F_{4}^{T} F_{8} C^{T}-\frac{1}{h} C F_{8}^{T} F_{19}\right), \\
\tilde{R}=\frac{1}{h} F_{30}^{T} F_{29} .
\end{gathered}
$$

Proof: The proof involves straightforward manipulations of matrix exponentials and hence is omitted.

\section{ACKNOWLEDGMENT}

The authors wish to thank Professor Steven R. Hall for pointing out the relevance of [11] to their results, Professor Pierre T. Kabamba for several helpful discussions, and the reviewer for pointing out an error in an earlier version of the note.

\section{REFERENCES}

[1] W. M. Haddad, D. S. Bernstein, H.-H. Huang, and Y. Halevi, "Fixedorder sampled-data estimation," Int. J. Contr., vol. 55, pp. 129-139, 1992.

[2] S. Shats and U. Shaked, "Exact discrete-time modelling of linear systems," Int. J. Contr., vol. 49, pp. 145-160, 1989.

[3] _ _, "Use of approximate discrete-time modeling in filtering and regulation of continuous-time processes," Int. J. Contr., vol. 50 , pp. 1297-1313, 1989.

[4] K. J. Astrom, Introduction to Stochastic Control Theory. New York: Academic, 1970.

[5] M. E. Polites and G. O. Beale, "Modelling and designing digital control systems with averaged measurements," Int. J. Contr., vol. 48, pp. 161-177, 1988.
[6] D. S. Bernstein, L. D. Davis, and S. W. Greeley, "The optimal projection equations for fixed-order sampled-data dynamic compensation with computation delay," IEEE Trans. Automat. Contr., vol. 31, pp. 859-862, 1986.

[7] D. S. Bernstein and W. M. Haddad, "Optimal reduced-order state estimation for unstable plants," Int. J. Contr., vol. 50, pp. 1259-1266, 1990.

[8] W. M. Haddad and D. S. Bernstein, "Optimal reduced-order subspaceobserver design with a frequency domain error bound," Adv. Contr. Dynam. Syst., vol. 31, C. T. Leondes, ed. New York: Academic, pp. 23-38, 1990.

[9] W. M. Haddad and D. S. Bernstein, "Optimal reduced-order observerestimators," AIAA J. Guidance Contr. Dyn., vol. 13, pp. 1126-1135 1990.

[10] S. Shats, B. Z. Bobrovsky, and U. Shaked, "Discrete-time state estimation of analog double integrators," IEEE Trans. Aero. Elec. Syst., vol. 24 , pp. $670-677,1988$.

[11] A. Gelb et al., Applied Optimal Estimation. Cambridge, MA: M.I.T. Press, 1974

[12] P. T. Kabamba, "Control of linear systems using generalized sampleddata hold functions," IEEE Trans. Automat. Contr., vol. AC-32, pp. 772-783, 1987.

[13] B. D. O. Anderson and J. B. Moore, "Time varying feedback laws for decentralized control," IEEE Trans. Automat. Contr., vol. AC-26, pp. 1133-1139, 1981

[14] S. H. Wang, "Stabilization of decentralized control systems via timevarying controllers," IEEE Trans. Automat. Contr., vol. AC-27, pp $741-744,1982$

[15] W. Sun, K. M. Nagpal, and P. P. Khargonekar, " $H_{\infty}$-control and filtering for sampled-data systems," preprint.

[16] W. M. Haddad and D. S. Bernstein, "The optimal projection equations for reduced-order state estimation: The singular measurement noise case," IEEE Trans. Automat. Contr., vol. 32, pp. 1135-1139, 1987.

[17] Y. Halevi, "The optimal reduced-order estimator for systems with singular measurement noise," IEEE Trans. Automat. Contr., vol. 34, pp. $777-781,1989$.

[18] H. Kwakernaak and R. Sivan, Linear Optimal Control Systems. New York: Wiley, 1972.

[19] C. F. Van Loan, "Computing integrals involving the matrix exponential," IEEE Trans. Automat. Contr., vol. 23, pp. 395-404, 1978.

[20] C. Moler, and C. F. Van Loan, "Nineteen dubious ways to compute the exponential of a matrix," SIAM Rev., vol. 20, pp. 801-836, 1978. 